\title{
CONTRADICCIONES DEL SIGNIFICADO DE LA ACTIVIDAD FÍSICO-DEPORTIVA EN LAS IDENTIDADES CORPORALES DE LAS CHICAS ADOLESCENTES
}

\author{
MARIA JOSÉ CAMACHO-MiÑANO \\ Universidad Complutense de Madrid
}

Recibido: 17/01/2013

Aceptado: 27/03/2013

\section{Resumen}

Dentro de la estructura de género que impone la construcción social del cuerpo femenino, el sentimiento de inadecuación del propio cuerpo parece caracterizar la configuración de las identidades corporales femeninas en la adolescencia, motivando que la relación que establecen muchas chicas jóvenes con la actividad físico-deportiva sea compleja y a menudo problemática. Siguiendo un enfoque feminista post-estructuralista, este trabajo se centra en tres conflictos: 1) La actividad física para perder peso, instrumentalizada dentro de los discursos para alcanzar el ideal de belleza o luchar contra la obesidad; 2) El límite socialmente construido para las chicas deportistas entre lo que se considera un tono muscular atractivo y un cuerpo excesivamente musculoso; 3) Los procesos de sexualización y ansiedad física social debido a la exposición del cuerpo a una mirada masculina no deseada en diferentes contextos de práctica físico-deportiva.

Palabras clave: mujeres adolescentes, feminidad, identidades, cuerpo, actividad física y deporte, post-estructuralismo.

\begin{abstract}
Within the gender structure imposed by the social construction of the female body, the feeling of inadequacy of one's body seems to characterize the configuration of female body identities in adolescence. This motivates among many young women a complex and often problematic relationship with physical activity and sport. Following a
\end{abstract}


post-structuralist feminist approach, this paper focuses on three conflicts: 1) Physical activity to lose weight within instrumentalized discourses to achieve the ideal of beauty as well as to fight against obesity, 2) The socially constructed limit for female athletes between what is considered an attractive muscle tone and excessive muscle development, 3) Sexualization and social physical anxiety due to body exposure to an unwanted male gaze in different contexts of physical activity and sport.

Keywords: Adolescent women, femininity, identities, body, physical activity and sport, post-structuralism. 


\section{Introducción}

A pesar de que los beneficios de la práctica de Actividad Física (en adelante, AF) en la edad adolescente están en la actualidad bien documentados ${ }^{1}$ resulta preocupante que una gran cantidad de jóvenes, particularmente chicas, no realicen el nivel de AF recomendado ${ }^{2}$ para obtener los beneficios que se derivan de la adopción de un estilo de vida activo. Por ejemplo, en el reciente estudio sobre los Hábitos Deportivos de la Población Escolar en España ${ }^{3} s e$ ha encontrado que solo el 31\% de las chicas realizan los niveles mínimos de AF necesarios para su salud.

Estos bajos niveles de AF de las chicas jóvenes descienden dramáticamente a lo largo de este período evolutivo siendo de reseñar, entre otros motivos, el fenómeno del abandono del deporte organizado y las actitudes poco positivas que muchas jóvenes manifiestan hacia la Educación Física escolar. La panorámica descrita acentúa la brecha de género existente, precisamente en una época en la que se sientan las bases de la futura participación en AF durante la edad adulta.

Es cierto que en nuestro país existen políticas e iniciativas institucionales orientadas a garantizar la igualdad en el ámbito del deporte y la $\mathrm{AF}^{4}$, pero la realidad de los datos y de las vivencias subjetivas de las jóvenes revelan que

1. JANSSEN, Ian \& LeBLANC, Allana G. Systematic review of the health benefits of physical activity and fitness in school-aged children and youth. International Journal of Behavioral Nutrition and Physical Activity 7, 1, (2010), pp. 40.

2. Se ha recomendado que la gente joven realice al menos 60 minutos de actividad física de intensidad moderada a vigorosa la mayoría de los días de la semana. WORLD HEALTH ORGANIZATION. Global recommendations on physical activity for health. Geneva, WHO, 2010. <http://whqlibdoc.who.int/publications/2010/9789241599979_eng.pdf >, consultado el 3-10-2010.

3. Este porcentaje de práctica de AF es de $54 \%$ en los chicos de la misma edad (6-18 años) y de sólo el 16\% de las chicas entre 16 y 18 años. Consejo Superior de Deportes.Los hábitos deportivos de la población escolar en España. Madrid, CSD, 2011.

4. Artículo 29. «Deportes», de la Ley Orgánica para la igualdad efectiva de mujeres y hombres (3/2007, de 22 de marzo).

Programa 9. «Igualdad efectiva entre mujeres y hombres», del Plan Integral para la Actividad Física y el Deporte (Plan A+D).Madrid, Consejo Superior de Deportes, 2010.

Feminismo/s 21, junio 2013, pp. 15-35 
siguen existiendo discursos y prácticas sociales que continúan reproduciendo el orden de género tradicional, limitando la participación y condicionando las experiencias de las chicas jóvenes en las actividades físico-deportivas.

En consecuencia, desde diversos organismos institucionales la población de chicas adolescentes ha sido identificada como un grupo prioritario necesitado de actuaciones específicas para la promoción de AF y para la consecución de la igualdad efectiva en los diversos contextos de práctica (Educación Física, deporte escolar, etc) ${ }^{5}$.

En el ámbito de la investigación ésta es una temática de máxima actualidad, si bien los resultados distan de ser alentadores ${ }^{6}$ y aún se desconoce con exactitud cómo conseguir que más chicas jóvenes adopten un estilo de vida activo y vivencien la AF y el deporte de forma positiva para la construcción de su identidad personal en esta etapa evolutiva.

Está claro que las razones de las chicas para enrolarse (o no) en la práctica de AF son complejas, pero más allá de una falta de motivación e interés personal hay que considerar que esta participación (o su ausencia) ocurren dentro de un contexto social y cultural fuertemente condicionado por la estructura de género, que reproduce los estereotipos tradicionales de masculinidad y feminidad y que moldea las experiencias y vivencias subjetivas en torno a la AF de forma poderosa.

Dentro de un orden de género cambiante y en constante transformación es de destacar cómo la construcción social del cuerpo femenino condiciona de un modo u otro la forma en que las chicas jóvenes negocian sus propias identidades corporales, lo que configura un panorama complejo y diverso en las relaciones que establecen las chicas jóvenes con la práctica de AFD. En consecuencia, la diversidad de subjetividades y múltiples identidades corporales de las jóvenes se posicionan ante los discursos dominantes, de forma que la vivencia del propio cuerpo en la práctica de actividad físico-deportiva puede ser fuente de empoderamiento, pero también de opresión y conflicto.

Desde esta perspectiva feminista post-estructuralista ${ }^{7}$, el presente artículo se centra en analizar el discurso en torno a la práctica de actividad físico-

5. EUROPEAN PARLIAMENT. «Resolution of 25 September 2008 on the White Paper on Nutrition, Overweight and Obesity- Related Health Issues (2007/2285(INI))». $<$ http://www.europarl.europa.eu/sides/getDoc.do? type=TA\&reference=P6-TA-20080461\&language $=\mathrm{EN}>$, consultado el 29-01-2011.

6. CAMACHO MiÑANO, Maria José, LaVOI, Nicole M., y BARR-ANDERSON, Daheia J. «Interventions to promote physical activity among young and adolescent girls: a systematic review». Health Education Research 26, 6 (2011), pp. 1025-1049.

7. Fundamentada en el trabajo de Foucault (1986) y aplicada por el feminismo de la tercera ola a la construcción social del cuerpo (p.e. Bordo, 1993a; Cole, 1994), esta perspectiva 
deportiva y el cuerpo femenino como construcción social, reflexionando de forma crítica sobre los conflictos y contradicciones para la configuración de las identidades corporales de las chicas adolescentes como mujeres físicamente activas.

\section{Actividad físico-deportiva, construcción social del cuerpo femenino e identidades corporales en la adolescencia}

La actividad física es un ámbito fundamental que condiciona y a su vez está mediatizado por la vivencia del propio cuerpo, puesto que la experiencia del movimiento implícita en toda acción motriz manifiesta la relación que cada individuo mantiene con su cuerpo. No obstante, esta relación no es aséptica sino que los diferentes significados identitarios que la práctica de $\mathrm{AF}$ adquiere para las chicas y para los chicos están fuertemente influenciados por la diferente valoración del cuerpo y los ideales de atractivo físico que se imponen como símbolos de la feminidad y la masculinidad ${ }^{8}$.

El poder de los discursos en torno al cuerpo y al género prescribe para las mujeres su identificación con un cuerpo objeto, en el que las diferentes partes corporales son evaluadas respecto al modelo ideal y por tanto, dependientes de la mirada del otro. En cambio, en la construcción de la masculinidad lo relevante es el valor de un cuerpo proceso, centrado en lo que se es capaz de conseguir mediante un uso activo del mismo. En ambos casos se trataría de una evaluación estereotípica del cuerpo (objeto/proceso, pasivo/activo), pero es indudable que la valoración de la mujer en base a su cuerpo como objeto implica una evaluación más negativa que como proceso, ya que su valía está sometida a la mirada de los demás y al criterio normativo de atractivo9.

En la actualidad esta posición dualista de los discursos sociales en torno a la valoración del cuerpo parece estar superada: son muchas las mujeres que se adscriben al concepto de cuerpo funcional a través de la práctica de $\mathrm{AF}$ y el culto al cuerpo también se ha convertido en una nueva exigencia para los varones dentro de la sociedad mercantilista actual donde el cuerpo se cotiza

ha hecho una contribución importante para romper con la definición bipolar de masculinidad-feminidad y fundamentar la diferencia con la existencia de múltiples feminidades y masculinidades debido a que las personas son sujetos activos en la construcción de su propia identidad de género.

8. Martínez Benlloch, Isabel, (coord.). Género, desarrollo psicosocial y trastornos de la imagen corporal. Madrid, Ministerio de Trabajo y Asuntos Sociales. Instituto de Estudios de la Mujer. Colección Estudios, 71, 2001.

9. PASTOR, Rosa. «Cuerpo y género: representación e imagen corporal», en Esther Barberá e Isabel Martínez (coords.): Psicología y Género, Madrid, Pearson Prentice Hall, 2004, pp. 217-240.

Feminismo/s 21, junio 2013, pp. 15-35 
como un objeto de consumo más ${ }^{10}$. Esta realidad pone de manifiesto la diversidad de identidades corporales y el diferente posicionamiento y negociación que hacen las personas de las ideologías corporales dominantes. Sin embargo, estas nociones estereotipadas en torno a la valoración del cuerpo todavía persisten e incluso parecen intensificarse en la edad adolescente. En este sentido es de reseñar el clásico estudio de Martínez Benlloch et al. ${ }^{11}$ que llegó a la conclusión de que los varones centraban su reconocimiento y autovaloración en variables relativas al autocontrol, poder personal, competencia y funcionamiento corporal, mientras que las mujeres adolescentes lo vinculaban a factores derivados de la conversión del cuerpo en objeto (preocupación por el peso, atractivo sexual y extrañamiento del cuerpo), explicándose así la mayor vulnerabilidad de las chicas a sufrir alteraciones de la imagen corporal.

Esta construcción social del cuerpo y del género motiva que la relación entre los chicos y la práctica de actividad física sea directa y sencilla, puesto que la masculinidad (hegemónica) ${ }^{12}$ se identifica con un cuerpo funcional, con la fuerza y competencia física. El deporte es un buen medio para reforzar esta masculinidad ya que es una manifestación que permite exhibir el cuerpo en acción, presentándolo ante los demás en una situación de competición y en el que la clave del éxito está en ser hábil motrizmente, agresivo y competitivo. Además, la participación deportiva refuerza el desarrollo muscular que se produce de forma natural con la llegada de la pubertad y que se impone como característica definitoria del ideal estético masculino. Así pues, incluso la identificación con una concepción de cuerpo objeto en su versión masculina resulta coherente y potencia de forma general la participación de los chicos jóvenes en actividades físico-deportivas ${ }^{13}$.

En cambio, dentro del orden de género establecido las relaciones de las chicas con la actividad físico-deportiva son mucho más complejas y, a menudo, conflictivas. Por una parte, el descenso de participación de las chicas adolescentes en la AF se ha atribuido a la reproducción de la feminidad, fuertemente influenciada por el entorno social y cultural ${ }^{14}$. Específicamen-

10. Featherstone, M. Consumer culture and postmodernism. Sage, London, 1991.

11. MARTíNEZ BENLlOCH, Isabel, Op. cit.

12. La existencia de otras identidades masculinas alternativas motiva que también haya chicos que no se identifican con este modelo de masculinidad hegemónica convirtiéndose también en identidades subordinadas dentro del orden de género dominante (Consúltese al respecto los trabajos de Raewyn CONNEL).

13. DitTmar, Helga, Lloyd, Barbara, DUGAN, Shaun, Halliwell, Emma, Jacobs, Neil y Cramer, Helen. «The Body Beautiful: English Adolescents' Images of Ideal Bodies». Sex Roles 42, 9-10 (2000), pp. 887-915.

14. CHOI, Precilla. Y. L. Femininity and the Physically Active Woman. New York, 2000. 
te, la práctica deportiva propiamente dicha parece ser incompatible con la feminidad tradicional en la edad adolescente; en palabras de las propias chicas: «no es femenino hacer deporte» ${ }^{15}$, si bien esta afirmación ha de ser matizada en función del tipo de deporte y de la tipología corporal que desarrolle su práctica. ${ }^{16}$

Por otra parte, la práctica de AF realizada por las chicas implica entroncar con un modelo de cuerpo funcional y activo que supone de un modo $\mathrm{u}$ otro romper las barreras de la feminidad. Pero entonces aparecen nuevas exigencias ya que tiende a compararse la competencia motriz de las chicas con un estándar de «deportista ideal» construido socialmente como masculino (fuerte, competitivo, agresivo). Se plantea en consecuencia un modelo de chica deportista «deficitaria», porque no se la valora en sí misma, por su individualidad, sino en función de su comparación con ese modelo masculino hegemónico ${ }^{17}$.

Aunque es una realidad que las chicas jóvenes se posicionan en relación a este orden de género y negocian su identidad corporal, oponiéndose, aceptando o incluso adaptando estos discursos sociales dominantes, lo cierto es que estos mensajes inciden de un modo u otro en la configuración de sus identidades, mediatizando de forma significativa las diferentes relaciones que las chicas establecen con las actividades físico-deportivas en los diferentes ámbitos de práctica ${ }^{18}$.

Es indudable que por los beneficios que reporta la AF sobre la salud de las jóvenes, así como por el hecho de que dicha práctica rompe a primera vista con el orden de género tradicional, resulta muy positivo que las chicas adolescentes sean capaces de asumir su cuerpo como entidad activa, capaz de actuar y conseguir logros relacionados con la práctica de actividad física. Esta afirmación general ha de ser matizada, analizada, reflexionada, debatida, puesto que en la relación entre actividad físico-deportiva, cuerpo femenino

15. Slater, Amy y Tiggemann, Marika «Uncool to do sport: A focus group study of adolescent girls' reasons for withdrawing from physical activity». Psychology of Sport $\&$ Exercise 11, 6 (2010), pp. 619-626.

16. El apartado 2.2. de este artículo aborda específicamente esta cuestión.

17. Este modelo de masculinidad hegemónica implica que todas las personas jóvenes que no se adscriben al mismo, y que son todas las chicas y muchos chicos con otras identidades masculinas alternativas a la tradicional, sufrirían este «déficit».

18. GARRET, Robyne. "Negotiation a physical identity: girls, bodies and physical education». Sport, Education and Society 9, 2, (2004), pp. 223-237.

AZZARITO, Laura. «Future girls, transcendent feminities and new pedagogies: toward girls' hybrid bodies». Sport, Education and Society 15, 3 (2010), pp. 261-275. 
adolescente e identidad confluyen muchas variables y discursos que es preciso visibilizar.

\section{Conflictos y paradojas en torno a la actividad físico-deportiva realizada por las adolescentes y la construcción social del cuerpo femenino}

Una mirada más profunda revela que la práctica de actividad física y deporte realizada por muchas chicas jóvenes encierra también paradojas importantes, puesto que determinadas prácticas y contextos de AF y deporte siguen perpetuando el orden de género establecido, reforzando las nociones hegemónicas de feminidad y dificultando el desarrollo de otras identidades corporales femeninas alternativas. A continuación se exponen tres fenómenos que tienen como eje la construcción social del cuerpo femenino y que resultan contradictorios e incluso conflictivos para la implicación de las adolescentes en las actividades físico-deportivas.

\subsection{Actividad física para perder peso: entre la búsqueda del ideal de belleza y la} lucha contra la obesidad

El énfasis en la pérdida de peso y tratar de ser delgada es un imperativo de la feminidad que caracteriza la cultura de las chicas jóvenes (y no tan jóvenes), convirtiéndose la AF en un medio para la consecución de la estética femenina definida como ideal.

La problemática principal radica en que esta delgadez que define al ideal femenino actual dista de corresponderse con los promedios naturales y anatómicos y, por tanto, es difícilmente alcanzable por la mayoría de la población femenina. Se trata de un ideal corporal irrealista y antinatural que choca con un modelo corporal basado en criterios normativos y de salud y que resulta particularmente conflictivo para las adolescentes, que deben asimilar cómo las transformaciones corporales propias de la pubertad (acumulación de grasa, ensanchamiento de caderas) las alejan cada vez más del referente cultural de cuerpo ideal.

Las chicas jóvenes negocian el sentido corporal de sí mismas y se posicionan de forma muy diversa en relación a este orden de género, pero es indudable que este mensaje en torno al ideal de delgadez se transmite de forma poderosa. La televisión, las revistas dirigidas a las adolescentes, los juguetes, los videojuegos, las películas de dibujos animados, etc. difunden este modelo corporal y sus supuestas bondades, de forma que las chicas jóvenes aprenden pronto el mensaje de la necesidad de ser atractiva y delgada para ser feliz. Los propios padres, el profesorado, el grupo de amigas y amigos, las entrenadoras/ 
es, etc., les transmiten mensajes implícitos y explícitos que conforman, a menudo de forma inconsciente, una pieza más del entramado que configura la «presión social» existente en torno al cuerpo ${ }^{19}$.

La posibilidad de conseguir el cuerpo femenino delgado se «vende» a través de toda una industria en torno al cuerpo, bajo la creencia de que el peso y la tipología corporal puede controlarse a voluntad, de forma que la persona puede elegir y es responsable de su tipología y peso corporal: solo con la correcta combinación de dieta y ejercicio cualquier persona puede alcanzar el ideal si se lo propone ${ }^{20}$. Se obvia así que el peso corporal, la distribución de grasa e incluso su porcentaje corporal tienen un fuerte componente hereditario y que las condiciones de vida que caracterizan a las sociedades «obesogénicas» ${ }^{21}$ promueven justamente la ganancia de peso.

Dentro de este contexto sociocultural muchas chicas realizan actividad física, motivadas principalmente por el deseo de mejorar su apariencia física, lo que reduce sus oportunidades de ver su cuerpo de una forma funcional y empoderada. Esta exigencia de ejercicio físico para conseguir el cuerpo ideal implica la existencia de ambigüedades y contradicciones, pues se dan diferentes ideas de feminidad en un mismo cuerpo que se resumen en la clásica expresión de Markula (1995): «delgado pero con curvas, en forma pero sexy, fuerte pero delgado» ${ }^{22}$.

Desde la perspectiva feminista post-estructuralista se ha señalado que la práctica de ejercicio físico para perder peso es una forma más de control sobre la mujer en cuanto que implica otro tipo de disciplina para conseguir el ideal de delgadez femenino, además de responder a la nueva exigencia de firmeza y tonificación ${ }^{23}$. La práctica de actividad física se realiza así con la idea de disciplinar el cuerpo antes que disfrutarlo. Además, la expectativa de

19. El Modelo Tripartito de Influencia Social explica la influencia de padres, iguales y media sobre el desarrollo de la imagen corporal a lo largo del desarrollo evolutivo. Un análisis sobre éste y otros aspectos relacionados, enfocados desde una perspectiva de género puede encontrarse en: CALOGERO, Rachel M. y THOMPSON, J. Kevin: «Gender and Body image», en Joan C. Chrisler y Donald.R. McCreary (Eds.): Handbook of Gender Research in Psychology. Vol II, 2010, pp. 153-184.

20. Rodin, Judith, SilberSTEIN, Lisa y STRIEGEL-MOORE, Ruth: «Women and weight: a normative discontent», en Theo B. Sonderegger (Ed.):Psychology and Gender: Nebraska Symposium on Motivation, Lincoln, University of Nebraska Press, 1984, pp. 267-307.

21. GraCiA-ARNaIZ, Mabel. «Fat bodies and thin bodies. Cultural, biomedical and market discourses on obesity». Appetite 55, 2, (2010), pp.219-225.

22. MARKULA, Pirkko. «Firm but shapely, fit but sexy, strong but thin: the postmodern aerobicizing female bodies». Sociology of Sport Journal 12 (1995), pp. 424-453.

23. BORDO, S. Unbearable weight: Feminism, Western culture, and the body. Berkeley, University of California Press, 1993b. 
delgadez refuerza el estatus subordinado de las mujeres en el orden de género y limita su potencial físico y social puesto que la persecución de este modelo de belleza, inalcanzable para la mayoría, implica un gasto considerable de tiempo, dinero y esfuerzo que limita los recursos personales de las mujeres para empoderarse $e^{24}$.

Paralelamente, aumentan las posibilidades de abandono de la propia práctica debido a que la AF para perder peso es una motivación extrínseca que llega a convertirse para algunas jóvenes en una obligación, reduciéndose la libertad percibida, la autonomía y el disfrute ${ }^{25}$. No obstante, la realización de ejercicio físico para mejorar la estética corporal puede suponer en ciertos casos el primer paso para la adhesión a una práctica más estable y regular que permita a estas jóvenes satisfacer sus necesidades, tanto estéticas como relacionadas con su salud y su sensación de bienestar. La clave se sitúa en cómo desplazar en estas chicas el foco de interés de la «representación» a la «acción» para conseguir que tengan una percepción más funcional de su cuerpo e incorporen la actividad física de forma saludable en su estilo de vida.

Este enfoque instrumental de la práctica de actividad física para perder peso también se defiende dentro del discurso en torno a la obesidad, que se ha señalado como alarmante en la población infantil y juvenil de la sociedad española y de otros países occidentales por sus riesgos para la salud. El problema se origina cuando se etiqueta como patológico un sobrepeso moderado, que en realidad no constituye un problema para la salud y la vida social, derivándose afirmaciones como «toda obesidad es perjudicial para la salud» y por tanto «la delgadez significa estar sano» y, más aún, «perder peso es saludable». De esta forma, el culto a la delgadez se transmite indirectamente a través de los discursos para actuar contra la obesidad. Además, se genera el estereotipo de que «lo gordo es feo», creencia que no se aplica por igual a los dos sexos, sino que se penaliza más para las mujeres. Se asocia «estar gorda» con ser fea y tener además un carácter débil y falta de control, mecanismos sociales de estigmatización que son interiorizados por el propio individuo, que acaba sintiéndose culpable, responsable y avergonzado de su propio cuerpo ${ }^{26}$.

DUNCAN, Margaret C. «The politics of women's body images and practices: Foucault, the panopticon, and shape magazine». Journal of Sport \& Social Issues 18, 1 (1994), pp. 48-65.

24. BORDO, S. Op. cit.

25. Craike, Melinda J., Symons, Caroline y Zimmermann, Jo An M. «Why do young women drop out of sport and physical activity? A social ecological approach». Annals of Leisure Research 12, 2 (2009), pp. 148-172.

26. GofFman, Erving. (Ed.). Estigma. La identidad deteriorada. Buenos Aires, Amorrortu, 1993. 
En definitiva, la delgadez se promueve para las chicas jóvenes desde discursos diferentes: ya sea para conseguir el ideal corporal femenino como baluarte de la belleza o bien para luchar contra la obesidad dentro de los esfuerzos de promoción de la salud. Y aunque se trata de orientaciones bien distintas, ambos discursos tratan de implicar a las chicas jóvenes en la AF a través de una aproximación instrumental, más que animar al disfrute y la motivación intrínseca ${ }^{27}$, enfoque éste último que facilitaría una adhesión más estable a la práctica física.

\subsection{Práctica del deporte y cuerpo tonificado / musculoso ¿dónde están los límites} de la feminidad?

La práctica del deporte organizado se ha vinculado en las chicas jóvenes con una vivencia más positiva del propio cuerpo $^{28}$, fundamentalmente porque centran prioritariamente la atención en lo que son capaces de hacer con su cuerpo y en cómo mejorar su competencia motriz para tener éxito en el deporte. Muchas mujeres deportistas consiguen a través de la vivencia de su cuerpo como entidad funcional romper con el ideal de feminidad tradicional vinculado con el cuerpo objeto. Pero por otra parte, existe la hipótesis de que esta imagen corporal más positiva se debe a que las deportistas consiguen desarrollar un cuerpo más delgado y tonificado gracias a la práctica regular de AF. De esta forma consiguen tener un aspecto físico más cercano a los cánones estéticos dominantes, enfoque que supondría una reproducción de la feminidad tradicional.

El tipo de deporte y su adscripción de género tradicional permiten explicar hasta qué punto las diferentes identidades corporales femeninas rompen o reproducen el orden de género dominante en torno a la representación del cuerpo. La distinción entre características y actividades femeninas y masculinas en el deporte corresponden a nociones estereotipadas basadas en cómo se construyen socialmente las diferencias entre las chicas y los chicos, más que basarse en diferencias reales. Existen así deportes tradicionalmente tipificados como masculinos, caracterizados por la fuerza, la resistencia, la agresividad (por ejemplo, fútbol, rugby, judo); y deportes femeninos, en los que resulta

27. RICH, Emma, EvANS, John \& PIAN, Laura: «Obesity, body pedagogies and young women's engagement with exercise», en Eileen Kennedy y Pirkko Markula (Eds.): Women and exercise: the body, health and consumerism, New York, Routedge, 2011, pp.138-158.

28. Camacho Miñano, Maria José, FernándeZ, Emilia, RodrígueZ, Ma Isabel (2006) «Imagen corporal y práctica de actividad física en las chicas adolescentes: incidencia de la modalidad deportiva». Revista Internacional de Ciencias del Deporte 3 (2006), pp.1-19.

Feminismo/s 21, junio 2013, pp. 15-35 
fundamental, entre otros aspectos, su componente estético (por ejemplo, natación sincronizada, patinaje artístico). Las diferencias de género en cuanto a cifras de participación en los diferentes deportes confirman en nuestra población adolescente la reproducción de este orden de género ${ }^{29}$.

La asociación entre género, forma y tipología corporal, y modalidad deportiva queda establecida, de forma que los deportes categorizados como femeninos se consideran como más apropiados para las chicas porque, entre otros aspectos, mediante su práctica se tienden a desarrollar cuerpos delgados y tonificados muy en consonancia con el ideal estético femenino. En cambio, aquellos deportes considerados tradicionalmente masculinos se consideran poco adecuados para ellas, en parte porque se asocian con la posibilidad de desarrollar demasiado músculo ${ }^{30}$.

Es indudable que dentro del deporte de rendimiento las chicas tienen que desarrollar cuerpos fuertes y musculosos para mejorar sus resultados deportivos. Pero el desarrollo muscular del cuerpo femenino que se consigue con la práctica de actividades físico-deportivas crea una paradoja: el cuerpo tonificado, duro, se percibe como ideal y deseable, pero los músculos excesivos simbolizan fuerza y masculinidad ${ }^{31}$. Algunas deportistas viven así con la contradicción de querer ser fuertes y tener éxito pero no desarrollar demasiado músculo. En realidad existe una línea arbitraria que demarca el límite entre lo que se considera un tono muscular atractivo (femenino) y un cuerpo excesivamente musculoso (masculino). Pasar este límite subjetivo y tener «demasiado músculo» es algo que socialmente no está bien visto y hace que estas chicas deportistas sean percibidas como «poco femeninas».

Las chicas practicantes de estos deportes y que desarrollan cuerpos musculosos desafían las estructuras de género, y por ello son penalizadas socialmente, puesto que transgreden la imagen de feminidad hegemónica (heterosexual) retando al poder y al privilegio masculino. Estas chicas deportistas se ponen en riesgo de ser acusadas de lesbianas, sintiéndose humilladas,

29. Existe también la categoría de deportes «neutros» que se consideran tradicionalmente como apropiados para ambos sexos. Un análisis sobre éste y otros aspectos relacionados puede consultarse en: RAMíREZ, Elena. Participación e implicación con la actividad físico-deportiva en la edad escolar. Un estudio de género desde la perspectiva socio-cognitiva. Tesis doctoral. Universidad Complutense de Madrid, 2010.

30. DUNCAN Margaret.C. «Sociological dimensions of girls' physical activity participation», en The 2007 Tucker Center Research Report, Developing Physically Active Girls: an Evidence-Based Multidisciplinary Approach. Minneapolis, MN, Tucker Center for Research on Girls \& Women in Sport, 2007, pp.29-50.

31. BORDO, S. Unbearable weight: Feminism, Western culture, and the body. Berkeley, University of California Press, 1993b. 
acosadas y discriminadas ante un discurso de homofobia y heterosexismo que puede apreciarse incluso en el ámbito de las clases de Educación Física ${ }^{32}$. La mujer excesivamente musculosa también puede ser acusada de consumo de esteroides $^{33}$, cuestionándose incluso su sexo biológico ${ }^{34}$.

Para negociar y reconciliar las expectativas sociales de feminidad con la práctica deportiva se ha señalado que las mujeres deportistas desarrollan una doble identidad: la de mujer y la de deportista, identidades que a veces confluyen y otras veces se mantienen separadas ${ }^{35}$. Por ejemplo, hay chicas deportistas satisfechas con su cuerpo deportivo pero que compensan su participación en el deporte y «reconstruyen» su yo femenino después de la práctica, maquillándose y arreglándose a toda prisa para parecer tradicionalmente femeninas. Incluso durante la competición deportiva, hay chicas que tratan de reforzar su feminidad (por ejemplo, con maquillaje, vestimenta «sexy», uñas pintadas, etc.).

Otras chicas deportistas viven con insatisfacción el desarrollo muscular, especialmente cuando se encuentran en otros contextos sociales en los que se valora tener un cuerpo cercano al modelo corporal femenino (por ejemplo, una fiesta); se produce así una contradicción entre su cuerpo «deportivo» y su cuerpo «social». Estos conflictos en torno al cuerpo deportivo femenino son resueltos por algunas jóvenes evitando implicarse en actividades físico-deportivas, como lo demuestra el hecho de que el miedo a desarrollar «demasiada» musculatura haya sido reconocido por las propias adolescentes como uno de los motivos por los que no hacen deporte ${ }^{36}$.

\section{3. «Puedo sentir cómo me miran»: ansiedad física social y sexualización en la actividad físico-deportiva}

La mayor parte de las actividades físico-deportivas que se desarrollan en los distintos contextos de práctica (Educación Física, deporte competición, etc.) tienen en común el hecho de realizarse en un entorno social público, lo que motiva que el cuerpo quede expuesto a la mirada evaluativa de los demás.

32. Sparkes, Andrew C., DeVIS, José y Fuentes, Miguel «iQué permanece oculto del currículum oculto? Las identidades de género y de sexualidad en la educación física». Revista Iberoamericana de educación, 39, (2005), pp. 73-90.

33. Lock, Rebecca Ann. «The Doping Ban: Compulsory Heterosexuality and Lesbophobia». International Review for the Sociology of Sport, 38, 4 (2003) pp. 397-411.

34. El caso más conocido es el de la corredora Caster Semenya.

35. Krane, Vikki, Choi, Precilla Y.L., Baird, Shannon M., Aimar, Christine M. \& Kauer, Kerrie «Living the Paradox: Female Athletes Negotiate Femininity and Muscularity». Sex Roles 50, 5-6 (2004), pp. 315-329.

36. Slater, Amy y Tiggemann, Marika. Op. cit.

Feminismo/s 21, junio 2013, pp. 15-35 
Debido al orden de género dominante, las mujeres están particularmente expuestas a la evaluación de su cuerpo como objeto, situación que se produce incluso cuando despliegan elevados niveles de competencia motriz. Esta realidad es particularmente evidente en la adolescencia, cuando las chicas jóvenes perciben que sus cuerpos son mirados, comentados y evaluados por otros debido a los cambios físicos puberales que experimentan ${ }^{37}$.

La problemática es que este proceso de objetivación o cosificación, es decir, de considerar su cuerpo como un objeto, es tan intenso que provoca que las chicas adolescentes lleguen a interiorizar las miradas presentes en las sociedad y empiecen a verse a sí mismas de la misma forma, es decir, adoptando la perspectiva de observador respecto a su físico y tratándose como un objeto para ser mirado y evaluado según su apariencia. Las chicas quedan así expuestas a la mirada panóptica que las vigila de posibles transgresiones contra los ideales patriarcales de feminidad a través de la monitorización privada y la autovigilancia ${ }^{38}$. Este proceso, denominado «auto-objetivación ${ }^{39}$, consiste en una forma de autoconciencia caracterizada por la monitorización constante de la propia apariencia física dando lugar a la aparición de numerosas consecuencias negativas. Entre ellas destacan el miedo y la vergüenza a que la propia apariencia física sea evaluada por otras personas, sufriendo lo que se conoce como ansiedad física social ${ }^{40}$.

Ciertos ambientes y actividades deportivas propician esa focalización en el cuerpo alentando identidades corporales conflictivas que explican determinados comportamientos en relación a la AF. El caso más representativo son las clases de aeróbic en las que normalmente hay espejos, se lleva ropa ajustada y existe competición entre las participantes sobre su aspecto físico ${ }^{41}$,

37. LindBer, Sara M., Grabe, Shelly, Hyde, Janet S. «Gender, pubertal development, and peer sexual harassment predict objectified body consciousness in early adolescence». Journal of Research on Adolescence» 17 (2007), pp. 723-742.

38. El concepto de poder panóptico de Foucault explica el poder positivo y funcional (en lugar de represivo) que va a motivar a los sujetos a interiorizar la vigilancia sobre su propio cuerpo. Autoras feministas como Pirkko MARKula y Margaret DUNCAN han aplicado esta perspectiva al ámbito de la actividad físico-deportiva realizada por las mujeres.

39. FREDRICKSON, Barbara. L., \& ROBERTS, Tommi-Ann. «Objectification theory - Toward understanding women's lived experiences and mental health risks». Psychology of Women Quarterly 21 (1997), pp. 173-206.

40. HARTER, S. «Causes, correlates, and the functional role of global self-worth: A life span perspective», en J. Kolligian yR. Stennberg (Eds.), Perceptions of competence and incompetence across the life span New Haven: Yale University Press, 1989, pp. 69-77.

41. Greenleaf, Christy., McGreer, Rosemary., \& Parham, Heather. «Physique Attitudes and Self-Presentational Concerns: Exploratory Interviews with Female Group Aerobic Exercisers and Instructors». Sex Roles 54, 3-4 (2006), pp. 189-199. 
lo que limita el disfrute con la práctica convirtiéndola en un elemento opresor que genera ansiedad, estrés e incluso métodos extremos de control de peso. En las clases de Educación Física se ha confirmado que hay chicas que se sienten incómodas en clases de natación experimentando una pobre imagen corporal situacional al sentir cómo sus cuerpos en bañador quedan expuestos a la mirada de los $\operatorname{chicos}^{42}$. Las chicas con identidades corporales más conflictivas optan por utilizar estrategias de afrontamiento, tanto de carácter cognitivo (tratando de auto-convencerse) como comportamental, minimizando la exposición del cuerpo o incluso poniendo excusas para evitar realizar la clase ${ }^{43}$.

También la situación de llevar uniformes deportivos que revelan el contorno corporal para competir provoca que las chicas se sientan sexualizadas, ya que sus cuerpos quedan expuestos y a menudo son el centro de atención de una mirada masculina no deseada. Incluso en algunos deportes (por ejemplo, voley playa), la indumentaria reglamentaria para competir es diferente para uno y otro sexo, quedando el cuerpo de las mujeres mucho más expuesto. Se trata de ropas que refuerzan la feminidad hegemónica y la mirada masculina, convirtiéndose así en otro instrumento que refuerza el orden de género al potenciar la imagen de mujer femenina heterosexual.

Esta misma imagen es la que acapara la representación de las mujeres deportistas en los medios de comunicación, imagen que se centra de forma reiterada en la apariencia femenina y el atractivo sexual de las deportistas, lo que conlleva implícitamente una desvalorización de su rendimiento deportivo $^{44}$. A pesar de que el problema de fondo en nuestro país sigue siendo

42. JAMES, Kandi. ««You can feel them looking at you»: the experiences of adolescent girls at swimming pools». Journal of Leisure Research, 32, 2 (2000), pp. 262-280.

43. ARAGÓN, Neila y CAMACHO-MiÑANO, Maria José. «Body image among adolescent girls in physical education swimming lessons».II Congreso Internacional de la Asociación Española de Ciencias del Deporte. Granada, 2012.

44. Messner, M. A. y COOKY, Cheryl (2010). Gender in Televised sports. News and highlighsts shows, 1989-2009.Center for Feminist Research, University of Southern California<http://dornsife.usc.edu/cfr/gender-in-televised-sports/>, consultado el 29-04-2013.

ROJAS TORRIJOS, José Luis. «La construcción de las noticias deportivas desde una mirada androcéntrica. De la invisibilidad a los estereotipos de la mujer deportista». Vivat Academia. $\mathrm{n}^{\circ} 113$ (2010) <http://www.ucm.es/info/vivataca/numeros/n113/DATOSS. htm>, consultado el 1-02-2011.

LÓPEZ DíEZ, Pilar (2011). Deporte y Mujeres en los medios de comunicación. Consejo Superior de Deportes: Madrid. <http://www.csd.gob.es/csd/estaticos/myd/web-guiammcc-vp.pdf>, consultado el 29-05-2012.

Feminismo/s 21, junio 2013, pp. 15-35 
la invisibilización de las deportistas en los $"$ media» $^{45}$, aquellas que reciben más atención son las que se adecuan a la feminidad hegemónica. Hay incluso algunas deportistas que llegan a posar desnudas para revistas masculinas y eligen esta sexualización autoimpuesta $\mathrm{o}$ "pornificación» ${ }^{46}$ para ganar, aparte de dinero, la atención de los medios de comunicación y el público (masculino). Estos mensajes tienen su impacto sobre las chicas deportistas adolescentes que aprenden que esta forma de feminidad hegemónica en el deporte tiene sus ventajas: aceptación social, privilegios (por ejemplo, apoyo de los sponsors) y, en definitiva, poder. Puesto que las mujeres deportistas son el modelo de referencia para las chicas más jóvenes, su sexualización resulta particularmente problemática ya que priva a las niñas y chicas adolescentes del valor intrínseco asociado a la práctica deportiva centrada en una concepción funcional del cuerpo. En consecuencia, este discurso de la sexualización en el deporte provoca que las jóvenes carezcan de una importante forma de resistencia ante el orden de género dominante.

\section{Conclusiones}

El sentimiento de inadecuación del propio cuerpo parece caracterizar la configuración de las identidades corporales femeninas en la adolescencia motivando que la relación que establecen las jóvenes con la actividad físico-deportiva sea compleja y a menudo conflictiva. Las causas son diversos: no percibirse lo suficientemente delgada, tener más músculo de lo que se considera un tono muscular femenino, o ser el centro de una mirada masculina no deseada.

Dentro de las demandas culturales que impone la construcción social del cuerpo femenino, las chicas adolescentes negocian los diferentes significados de la práctica de actividad físico-deportiva y configuran de forma activa identidades corporales diversas, ya sea resistiendo, reproduciendo o adaptando los discursos dominantes sobre la feminidad.

45. El CONSEJO AUDIOVISUAL DE ANDALUCÍA (2008)confirmó que del total de emisiones dedicadas en televisiones nacionales y regionales a eventos deportivos solo un $5 \%$ se refería a deportes protagonizados por mujeres.

46. JIRASEK, Ivo., KOHE, Geoffery Zain, \& HURYCH, Emanuel. «Reimagining athletic nudity: the sexualization of sport as a sign of a 'porno-ization' of culture». Sport in Society: Cultures, Commerce, Media, Politics (2013). Anticipo de publicación en línea: DOI:10. 1080/17430437.2012.753525.

La revista Interviú «Desnuda a deportistas olímpicas» (Julio de 2012) es el caso más reciente y llamativo en nuestro país. 
En este contexto es fundamental el desarrollo de una pedagogía corporal ${ }^{47}$ que favorezca en las chicas jóvenes otras formas de vivenciar su cuerpo y la práctica de actividad físico-deportiva. La clave se sitúa en conseguir que dicha práctica se convierta en un ámbito más para la realización personal, que dé respuesta a las necesidades e intereses personales de las chicas jóvenes y que contribuya a la construcción de una identidad propia, rompiendo las estrechas nociones de feminidad. Para ello es fundamental, entre otros aspectos, el desarrollo del conocimiento de uno mismo, facilitando espacios y oportunidades que permitan «deconstruir» las categorías de género dominantes, mediante el análisis de los discursos sociales que influyen en la producción de la feminidad.

Dentro de un marco post-estructuralista, esta pedagogía corporal debería tener en cuenta el contexto específico de las diferentes prácticas de actividad físico-deportiva así como la compleja articulación que se produce entre el género y otras variables como la edad, la raza o la clase social y que da lugar a entidades corporales aún más diversas.

Finalmente, en nuestro contexto sociocultural resulta crucial que las personas que trabajan con las chicas jóvenes (personal técnico deportivo, profesorado de Educación Física) reciban la formación ${ }^{48}$ necesaria para comprender el influjo del orden de género dominante sobre los significados subjetivos del propio cuerpo en estas edades. De esta forma, podrían contribuir a proporcionar experiencias motrices satisfactorias que respeten y potencien la construcción de una identidad personal propia en la que confluyan de forma positiva ser mujer adolescente y físicamente activa.

47. RICH, Emma, EvANS, John \& PIAN, Laura: «Obesity, body pedagogies and young women's engagement with exercise», en Eileen Kennedy y Pirkko Markula (Eds.): Women and exercise: the body, health and consumerism, New York, Routedge, 2011, pp.138-158.

WRIGHT, Janice E. «Biopower, biopedagogies and the obesity epidemic», en J. Wright $\&$ V. Harwood (Eds.), Biopolitics and the Obesity Epidemic: goberning bodies, New York: Routedge, 2009, pp. 1-14.

Dentro del contexto español véanse p.e., los trabajos de VAZQUEZ, Benilde (2001). «Los valores corporales y la Educación Física: hacia una reconceptualización de la Educación Física». Ágora para la EF y el Deporte, 1 (2001), pp. 7-17.

48. WRIGHT, Jane. «Physical Education Teacher Education: sites of progressor resistance», en D. Penney (Ed.), Gender and Physical Education,London: Routledge, 2002, pp. 190-207.

Véanse como propuestas de aplicación práctica para que el profesorado de Educación Física trabaje esta temática con las jóvenes los estudios de Oliver y Lalik (2004) y O'Brien et al. (2008).

Feminismo/s 21, junio 2013, pp. 15-35 


\section{Referencias bibliográficas}

ARAGÓN, Neila y CAMACHO-MiÑANO, Maria José. «Body image among adolescent girls in physical education swimming lessons».II Congreso Internacional de la Asociación Española de Ciencias del Deporte.Granada, 2012.

AzZARITO, Laura. «Future girls, transcendent feminities and new pedagogies: toward girls' hybrid bodies». Sport, Education and Society, 15, 3 (2010), pp. 261-275.

BORDO, S. Feminism, Foucault and the politics of the body. En C. Ramazanoglu (Ed.): Up against Foucault. Exploration of some tensions between Foucault and feminism. New York, Routledge, 1993a.

BORDO, S. Unbearable weight: Feminism, Western culture, and the body. Berkeley, University of California Press, 1993b.

CAmacho-Miñano, Maria José, Lavor, Nicole y BaRR-Anderson, Daheia. «Interventions to promote physical activity among young and adolescent girls: a systematic review». Health Education Research 26, 6 (2011), pp. 1025-49.

Camacho Miñano, Maria José, Fernández, Emilia, Rordríguez, Ma Isabel. «Imagen corporal y práctica de actividad física en las chicas adolescentes: incidencia de la modalidad deportiva». Revista Internacional de Ciencias del Deporte 3 (2006), pp.1-19.

CHOI, Precilla. Y. L. Femininity and the Physically Active Woman. New York, Routledge, 2000.

COCKBURN, Claudia y CLARKE, Gill. «Everybody's looking at you!»: Girls negotiating the "femininity deficit» they incur in physical education». Women's Studies International Forum 25, 6 (2002), pp. 651-665.

COLE, Cherry L. «Resisting the canon: Feminist cultural studies, sport, andtechnologies of the body», en S. BirRell \& C.L. COLE (Ed.):Women, sport, and culture. Human Kinetics, Champaign,1994, pp. 5-29.

CONSEJO AUDIOVISUAL DE ANDALUCÍA (2008). Estudio sobre género y deporte en televisión.CAA.<http://www.consejoaudiovisualdeandalucia.es/ sites/default/files/publicaciones/estudio_sobre_gxnero_y_deporte_en_ televisixn_2008.pdf>, consultado el 29-03-2013.

CONSEJO SUPERIOR DE DEPORTES. Los hábitos deportivos de la población escolar en España. Madrid, CSD, 2011.

CONSEJO SUPERIOR DE DEPORTES. Plan Integral para la Actividad Física y el Deporte (Plan $\mathrm{A}+\mathrm{D})$.Programa 9. «Igualdad efectiva entre mujeres y hombres», Madrid, 2010.

Craike, Melinda J., Symons, Caroline y Zimmermann, Jo An M. «Why do young women drop out of sport and physical activity? A social ecological approach». Annals of Leisure Research 12,2 (2009), pp. 148-172. 
DitTmar, Helga, Lloyd, Barbara, Dugan, Shaun, Halliwell, Emma, Jacobs, Neil y Cramer, Helen. «The Body Beautiful: English Adolescents' Images of Ideal Bodies». Sex Roles 42, 9-10 (2000), pp. 887-915.

DUNCAN, Margaret C. «The politics of women's body images and practices: Foucault, the panopticon, and shape magazine». Journal of Sport E Social Issues 18, 1 (1994), pp. 48-65.

DUNCAN, Margaret C. «Sociological dimensions of girls' physical activity participation», en The 2007 Tucker Center Research Report, Developing Physically Active Girls: an Evidence-Based Multidisciplinary Approach. Minneapolis, MN, Tucker Center for Research on Girls \& Women in Sport, 2007, pp.29-50.

HARTER, S. Causes, correlates, and the functional role of global self-worth: A life span perspective. En J. Kolligian yR. Stennberg (Eds.): Perceptions of competence and incompetence across the life span. New Haven, Yale University Press, 1989, pp. 69-77.

EUROPEAN PARLIAMENT. «Resolution of 25 September 2008 on the White Paper on Nutrition, Overweight and Obesity - Related Health Is-

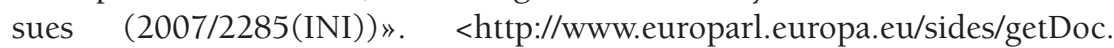
do type $=$ TA\&reference $=$ P6-TA-2008-0461 $\&$ language $=E N>$, consultado el 29-01-2011.

Featherstone, Mike. Consumer culture and postmodernism. Sage, London, 1991.

FOUCAUlT, Michael. Vigilar y castigar. Siglo XXI, Madrid, 1986.

FREDRICKSON, Barbara. L., \& ROBERTS, Tommi-Ann. «Objectification theory Toward understanding women's lived experiences and mental health risks». Psychology of Women Quarterly 21 (1997), pp. 173-206.

GARRET, Robyne. «Negotiation a physical identity: girls, bodies and physical education». Sport, Education and Society 9, 2, (2004), pp. 223-237.

Goffman, Erving. (Ed.): Estigma. La identidad deteriorada. Buenos Aires, Amorrortu, 1993.

GRACIA-ARNAIZ, Mabel. «Fat bodies and thin bodies. Cultural, biomedical and market discourses on obesity». Appetite 55, 2, (2010), pp. 219-225.

Greenleaf, Christy, McGreer, Rosemary., \& PARHAM, Heather. «Physique Attitudes and Self-Presentational Concerns: Exploratory Interviews with Female Group Aerobic Exercisers and Instructors». Sex Roles 54, 3-4 (2006), pp. 189-199.

JAMES, Kandi. ««You can feel them looking at you»: the experiences of adolescent girls at swimming pools». Journal of Leisure Research 32, 2 (2000), pp. 262-280.

JANSSEN, Ian \& LEBLANC, Allana G. «Systematic review of the health benefits of physical activity and fitness in school-aged children and youth». International Journal of Behavioral Nutrition and Physical Activity 7, 1, (2010), pp. 40. 
JiRASEK, Ivo, KoHE, Geoffery Zain, \& HURYCH, Emanuel. Reimagining athletic nudity: the sexualization of sport as a sign of a 'porno-ization' of culture. Sport in Society: Cultures, Commerce, Media, Politics (2013). Anticipo de publicación en línea: DOI:10.1080/17430437.2012.753525.

Krane, Vikki, Choi, Precilla Y.L., BAIRd, Shannon M., Aimar, Christine M. \& KAUER, Kerrie «Living the Paradox: Female Athletes Negotiate Femininity and Muscularity». Sex Roles 50, 5-6 (2004), pp. 315-329.

LEY ORGÁNICA para la igualdad efectiva de mujeres y hombres (3/2007, de 22 de marzo).

LindBer, Sara M., Grabe, Shelly, Hyde, Janet S. «Gender, pubertal development, and peer sexual harassment predict objectified body consciousness in early adolescence». Journal of Research on Adolescence» 17 (2007), pp. 723-742.

Lock, Rebecca Ann. «The Doping Ban: Compulsory Heterosexuality and Lesbophobia». International Review for the Sociology of Sport 38, 4 (2003), pp. 397-411.

LÓPEZ DíEz, Pilar (2011). Deporte y Mujeres en los medios de comunicación. Consejo Superior de Deportes: Madrid. <http://www.csd.gob.es/csd/estaticos/ $\mathrm{myd} /$ web-guia-mmcc-vp.pdf $>$, consultado el 29-05-2012.

MARKUlA, Pirkko. «Firm but shapely, fit but sexy, strong but thin: the postmodern aerobicizing female bodies». Sociology of Sport Journal 12 (1995), pp. 424-453.

MARTínez Benlloch, Isabel, (coord.). Género, desarrollo psico-social y trastornos de la imagen corporal. Madrid, Ministerio de Trabajo y Asuntos Sociales. Instituto de Estudios de la Mujer. Colección Estudios 71, 2001.

Messner, M. A. y CoOky, Cheryl (2010). Gender in Televised sports. News and highlighsts shows, 1989-2009. Center for Feminist Research, University of Southern California. <http://dornsife.usc.edu/cfr/gender-in-televisedsports/>, consultado el 29-03-2013.

Oliver, Kimberly L. \& LALIK, Rosary. «Critical Inquiry on the Body in Girls' Physical Education Classes: A Critical Poststructural Perspective». Journal of Teaching in Physical Education 23 (2004), pp. 162-195.

O'BriEn, Jacinta, MARTIN, Kathleen A., KIRK, David. «The effects of a body-focused physical and health education module on self-objectification and social physical anxiety in Irish girls». Journal of Teaching in Physical Education 27, 1, (2008), pp.116-125.

PASTOR, Rosa. «Cuerpo y género: representación e imagen corporal», en Esther Barberá e Isabel Martínez (coords.): Psicología y Género. Madrid, Pearson Prentice Hall, 2004, pp. 217-240.

RAMíREZ, Elena. Participación e implicación con la actividad físico-deportiva en la edad escolar. Un estudio de género desde la perspectiva socio-cognitiva. Tesis doctoral. Universidad Complutense de Madrid, 2010. 
RICH, Emma, Evans, John \& PIAN, Laura: «Obesity, body pedagogies and young women's engagement with exercise», en Eileen Kennedy y Pirkko Markula (Eds.): Women and exercise: the body, health and consumerism, New York, Routedge, 2011, pp.138-158.

RODIN, Judith, SILBERSTEIN, Lisa y STRIEGEL-MOORE, Ruth: Women and weight: a normative discontent», en Theo B. Sonderegger (Ed.):Psychology and Gender: Nebraska Symposium on Motivation,Lincoln, University of Nebraska Press, 1984, pp. 267-307.

ROJAS TORRIJOS, José Luis. «La construcción de las noticias deportivas desde una mirada androcéntrica. De la invisibilidad a los estereotipos de la mujer deportista». Vivat Academia. no 113 (2010) <http://www.ucm.es/info/vivataca/ numeros/n113/DATOSS.htm>, consultado el 1-02-2011.

Slater, Amy y Tiggemann, Marika «Uncool to do sport: A focus group study of adolescent girls' reasons for withdrawing from physical activity». Psychology of Sport E Exercise 11, 6 (2010), pp. 619-626.

SPARKeS, Andrew C., DeVIS, José y FUENTES, Miguel «¿Qué permanece oculto del currículum oculto? Las identidades de género y de sexualidad en la educación física», Revista Iberoamericana de educación 39, (2005), pp. 73-90.

VÁZQUEZ, Benilde. «Los valores corporales y la Educación Física: hacia una reconceptualización de la Educación Física». Ágora para la EF y el Deporte 1 (2001), pp. 7-17.

WORLD HEALTH ORGANIZATION. Global recommendations on physical activity for health. Geneva, WHO, 2010.

WRIGHT, Jane. «Physical Education Teacher Education: sites of progress or resistance», en D. Penney (Ed.), Gender and Physical Education, London: Routledge, 2002, pp. 190-207.

Wright, Janice E. «Biopower, biopedagogies and the obesity epidemic», en J. Wright \& V. Harwood (Eds.), Biopolitics and the Obesity Epidemic: goberning bodies, New York: Routedge, 2009, pp. 1-14. 